\title{
Secondary students' cognitive processes for the line graph from graph components
}

\author{
Tae-Sun Kim and Beom-Ki Kim \\ Dept. of Physics Education, Korea National University of Education, Cheongwon, 363-791, Korea
}

Line graphs are frequently used to communicate data and basic concepts in classroom activities; unfortunately, little has been reported concerning the students' cognitive process regarding line graphs. This study was intended to investigate such cognitive process empirically. We developed a computer program to determine the order readers glance the components of a line graph. We analyzed the glancing order of each component. The results help us identify secondary students' cognitive process for line graph.

\section{INTRODUCTION}

Line graphs are an important facet in physics education. Graphing abilities are critical to understanding and conveying information in physics. So, we wondered to what extent are secondary students in physics courses able to understand line graphs? Unfortunately, researchers find a widespread lack of graphing abilities ${ }^{1}$. Although the use of graphs becomes common in even primary school, many secondary students are weak in the ability to construct and interpret global graphical features ${ }^{2}$. In addition, we know relatively little about the nature of these points $^{3}$. And until now we have not known the specific cognitive operations upon which this graphing ability is based.

The purpose of this study is to find clues about secondary students' cognitive processes regarding line graphs: Is there a statistically significant order and a statistically significant difference between the viewing times of each component of a line graphs?

\section{METHODS}

We first investigated the typical line graph found in many science textbooks in Korea. We found that most graphs are line graphs and they assume the form shown in Figure 1. (For more information, see Kim \& Kim, 2001. $)^{4}$

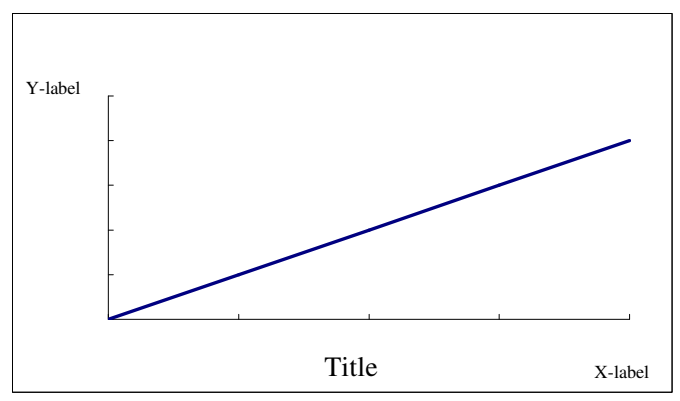

Figure 1 The typical line graph's shape in Korean textbooks

Next we developed a computer program written in Visual Basic. At first, a graph is displayed with seven buttons covering key parts of the graph. Each button opens whenever a participant clicks on it. To avoid misunderstanding, we explain to the student on the screen how the program works. When the student chooses a component the program records which component was chosen.

Figure 2 shows a screen shoot seen by a student when the program is first run. All 
seven components (Title, X-scale, Y-scale, Xlabel, Y-label, Data region, Legend) are covered by jacket. Subjects open any component they want when they answer the accompanying question. Figure 3 shows a sample of a subject who has opened two components (Y-scale and Data region).

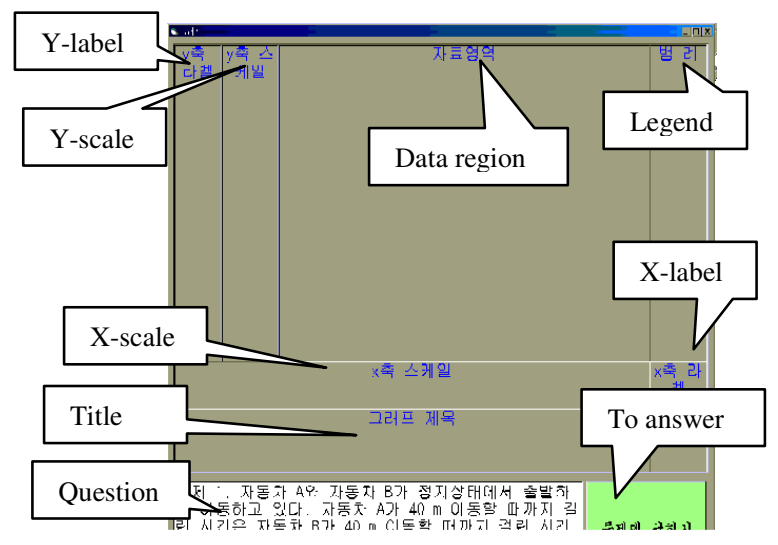

Figure 2 A sample item is covered by jacket

At the two high schools, 45 students (25 females and 20 males) and 16 students (16 males) participated in this study, respectively.

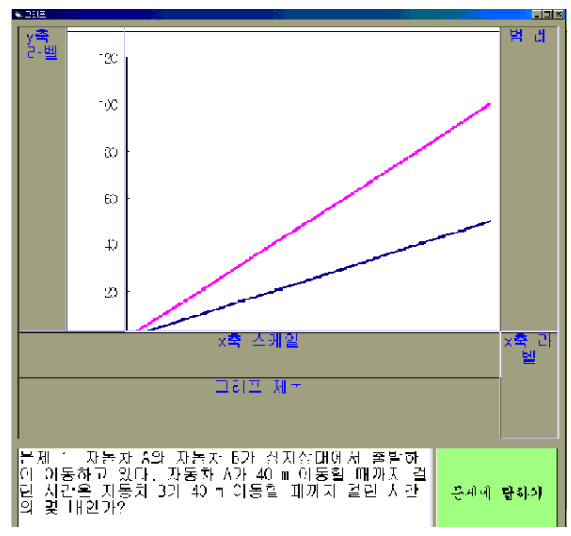

Figure 3 A sample item with two components (Y-scale and Data region) opened by a subject

\section{RESULTS}

We analyzed the data gathered by the program by looking at viewing order each component of a line graph.

Table 1 gives the order in which subjects choose to read the seven graph components. The total number of combinations is 610 , for there are 10 line graphs $\times 61$ subjects. To verify whether or not there was any difference in the viewing order of each component, we analyzed the results using Chi square analyses. Chi square analyses revealed that graph components were selected in a statistically significant order $\left[X^{2}=413.87, \mathrm{p}<.05\right]$.

Table 1 The numbers of occasions according to the order in which participants choose to view the seven graphing components

\begin{tabular}{|c|c|c|c|c|c|c|c|}
\hline \multicolumn{8}{|c|}{ Viewing order } \\
\hline & $1 \mathrm{st}$ & 2nd & $3 \mathrm{rd}$ & 4th & 5th & 6th & 7th \\
\hline & $\mathrm{N}(\%)$ & $\mathrm{N}(\%)$ & $\mathrm{N}(\%)$ & $\mathrm{N}(\%)$ & $\mathrm{N}(\%)$ & $\mathrm{N}(\%)$ & $\mathrm{N}(\%)$ \\
\hline Title & $23(3.8)$ & $19(3.1)$ & $53(8.7)$ & $53(8.7)$ & $205(33.6)$ & $65(10.7)$ & $192(\mathbf{3 1 . 5})$ \\
\hline Y-label & $205(33.6)$ & $37(6.1)$ & $163(26.7)$ & $41(6.7)$ & $87(14.3)$ & $12(2.0)$ & $65(10.7)$ \\
\hline X-label & $2(0.3)$ & $1(0.2)$ & $19(3.1)$ & $24(3.9)$ & $190(\mathbf{3 1 . 1})$ & $206(33.8)$ & $168(\mathbf{2 7 . 5})$ \\
\hline Y-scale & $35(5.7)$ & $386(63.3)$ & $73(12.0)$ & $65(10.7)$ & $4(0.7)$ & $46(7.5)$ & $1(0.2)$ \\
\hline X-scale & $32(5.2)$ & $96(15.7)$ & $55(9.0)$ & 197(32.3) & $68(11.1)$ & $141(\mathbf{2 3 . 1})$ & $21(3.4)$ \\
\hline Data region & $312(51.1)$ & $36(5.9)$ & $233(38.2)$ & $3(0.5)$ & $23(3.8)$ & $0(0.0)$ & $3(0.5)$ \\
\hline Legend & $1(0.2)$ & $35(5.7)$ & $14(2.3)$ & $227(37.2)$ & $33(5.4)$ & $140(\mathbf{2 3 . 0})$ & $160(\mathbf{2 6 . 2})$ \\
\hline Total & 610 & 610 & 610 & 610 & 610 & 610 & 610 \\
\hline
\end{tabular}




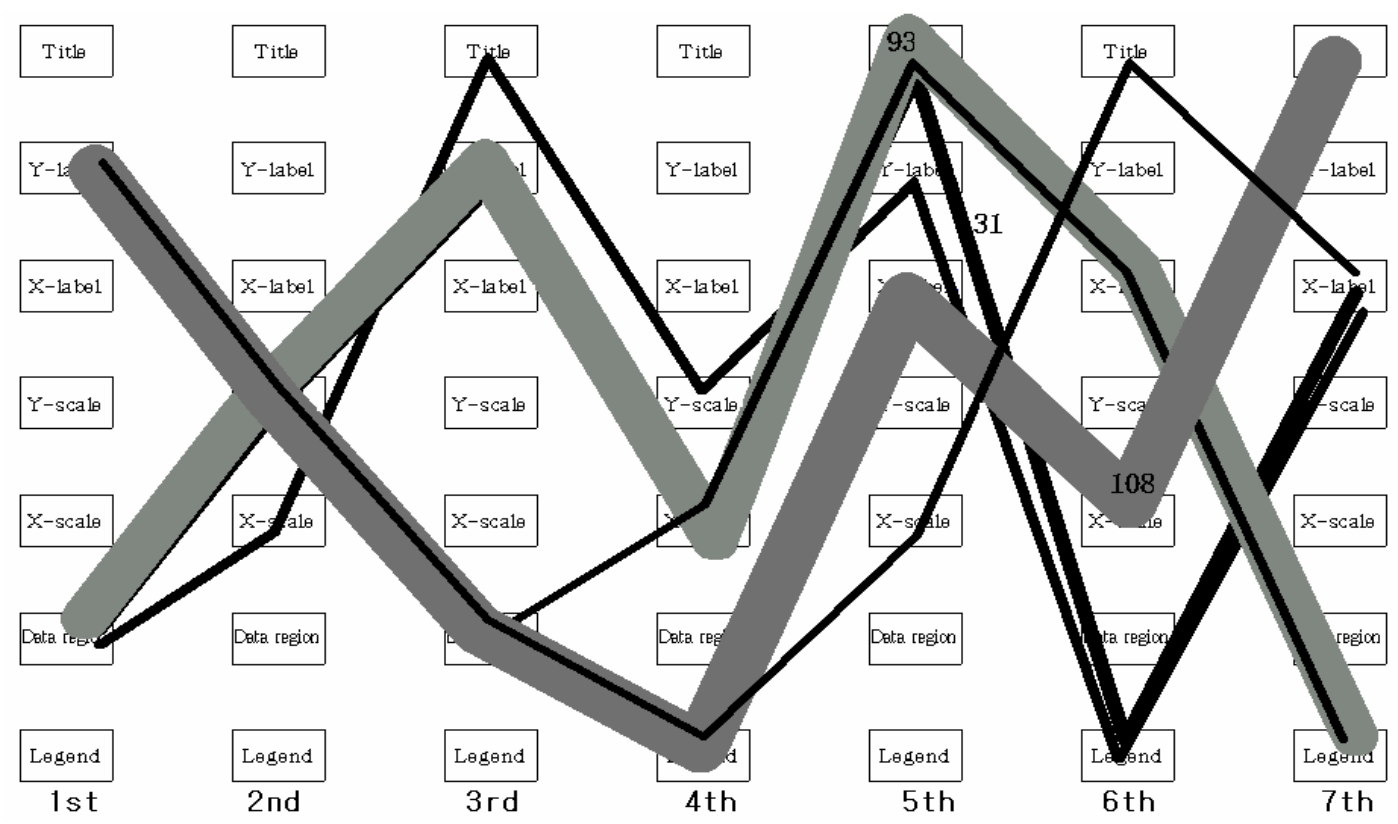

Figure 4 the order that each student clicked the graphing components

"Data Region" was clicked first among the majority of trials $(51.1 \%)$, followed by "Y-label" (33.6\%). The other components were viewed less frequently initially. There was a tendency for the "Title" to be selected last. This means that many secondary students are initially concerned more about the data region or the dependent variable (i.e., the Y-label and the Y-scale) before other components.

Figure 4 shows some of the orders in which students clicked the graphing components. The heavier the line, the more students clicked on that combination. Using Figure 4, we can make the possible viewing route (Figure 5) gained by typical readers in Korea.

We found that they have almost two typical orders to read each graph component. One is this: Y-label, Y-scale, Data region, Legend, X-label, X-scale, and Title in the

\section{CONCLUSION}

The most frequent last combination was "Legend" followed by the "Title". (See Figure

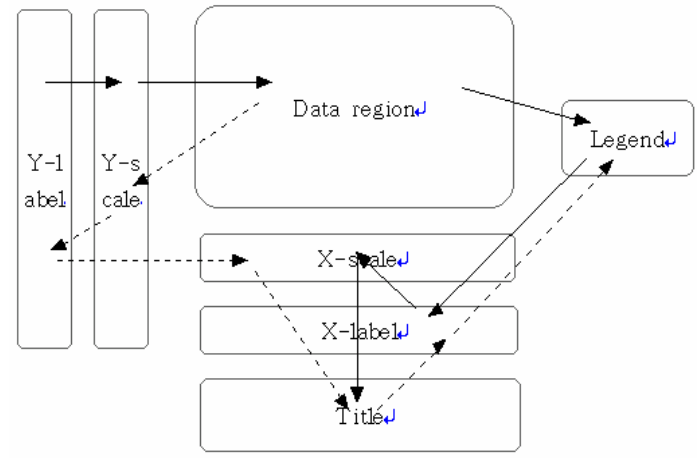

Figure 5 viewing orders of typical participants

order named. The other is this: Data region, Y-scale, Y-label, X-scale, X-label, Title, and Legend in the order named. What is the difference between two orders is further study. 4.) This makes senses because the title function as a clue to the whole image of a line graph. So then, why don't they read the title first? Where did the tendency, to look at the "Title" first, come from? There are at least two points of view about that. One is that 
participants might be influenced by Korean cultural customs when text is read from top to bottom and from left to right. In our study, we placed the "Title" on the bottom of the line graph, same as the system of the line graphs in Korean textbooks. The other is the function of the title that, at least our students thought, might be a mere scrap of paper nowadays. So it couldn't express the content of the line graph any more. The Titles of line graphs in Korean textbooks are single brief sentences that give no details, while graphs in scientific journal articles provide many resources to assist readers and direct readers' attention. So, it may possibly help readers.

Line graphs are efficient tools for making sense of information. They are used as an integral part of information transfer in many contexts of physics education. So it is important that they be become cognitively available to students in many courses. We gained an insight into the cognitive strategies students use to read line graphs by investigating the viewing order and the time spent reading each component of a line graph. We believe this study provides a way to explore students' cognitive processes regarding the line graphs.

We investigated any difference between the lengths of time subjects spent studying each of the seven components by our program, as well as the order of component selection. For more information about this study and what means the time subjects spent studying each component, see Kim \& Kim, 2001.

${ }^{1}$ C. A. Berg and D. G. Phillips, "Investigation of the relationship between logical thinking structures and the ability to construct and interpret line graphs," Journal of Research in Science Teaching, 31, 323-344 (1994).

${ }^{2}$ U. Maichle, "Cognitive processes in understanding line graphs," in W. Schnotz and R. W. Kulhavy (Eds.), "Comprehension of Graphics," 207-226, Elsevier Science B. V. (1994).

${ }^{3}$ R. W. Jones, J. W. Warner, and S. G. Fankhauser, "Investigating student understanding of graphs: A successful methodology and results of a study," Paper presented at the Conference of the National Association for Research in Science Teaching, Boston (1999).

${ }^{4}$ T. S. Kim, E. M. Kim and B. K. Kim, "Posing students' cognitive processes for the line graph," Paper presented at the annual meeting of the International Conference on Physics Education in Cultural context (ICPEC) (2001). 